\title{
On the Periodic Solutions of the Davey-Stewartson Systems
}

\author{
Gino Biondini* and Dmitri Kireyev \\ Department of Mathematics, State University of New York, Buffalo, NY 14260, USA.
}

Received 31 March 2021; Accepted (in revised version) 23 August 2021.

\begin{abstract}
The periodic, traveling wave solutions of all four versions of the DaveyStewartson system (namely the focusing and the defocusing cases of both the DaveyStewartson I and the Davey-Stewartson II equations) are derived and classified. For all four versions, these solutions are described in terms of elliptic functions. Special reductions and limiting cases, including harmonic limits, soliton limits, and one-dimensional solutions, are also explicitly discussed.
\end{abstract}

AMS subject classifications: 35Q51, 35B10, 35C08, 37K10

Key words: Davey-Stewartson, periodic solutions, solitons, elliptic functions.

\section{Introduction and Background}

Equations of nonlinear Schrödinger (NLS) type arise as physical models in a number of different physical contexts, ranging from water waves, to optics, Bose-Einstein condensates, plasmas and more $[11,31,35,39]$. The simplest and most well-known case is of course that of the cubic NLS equation itself in one spatial dimension. There are also many physical contexts, however, in which the system is not confined to just one coordinate, and two spatial dimensions are necessary to accurately describe the dynamics. In these situations, more general systems of equations of NLS type often arise, in which the dynamics of an NLS-type weakly nonlinear envelope to that of a "mean field" $[10,14,54]$. One such case is that of the equations that model the evolution of wave packets in shallow water [10], a special limit of which gives rise to the Davey-Stewartson system [20]. Similar systems of NLS-type equations with coupling to mean fields have also been derived in optical materials with quadratic nonlinearity $[1,2]$.

Like the NLS equation $[56,57]$, the Davey-Stewartson system of equations is also an integrable system [6]. As such, it possesses a deep mathematical structure - cf. [6], including the existence of a Lax pair, the Painlevé property $[8,9,47,49]$, the amenability of its initial value problem to inverse scattering $[26,27]$, the existence of a rich family of solutions,

${ }^{*}$ Corresponding author. Email addresses: biond ini@buffalo.edu (G. Biondini), dmitriki@buffalo.edu (D. Kireyev) 
including solitons as well as exponentially localized objects called dromions [17,28], even more exotic solutions [48] and more. Because of this, the Davey-Stewartson system continues to a very active topic of study $[12,29,36,37,42,52,53]$. Nonlocal variants of the DaveyStewartson system have also been a subject of study in recent years - cf. [25, 43, 44, 50] and references therein.

Like most other integrable evolution equations, the one-dimensional NLS equation also admits a rich family of traveling wave periodic solutions [13,32-34], which are expressed in terms of elliptic functions. The well-known soliton solutions are simply the limiting case of this more general family of solutions. In turn, these solutions provide the starting point for further investigations such as stability $[18,19,21]$ as well as dispersionless or semiclassical limits $[12,36,37]$. It would be safe to expect that a similar class of solutions also exists for the Davey-Stewartson system. Surprisingly, however, no such solutions have been presented in the literature to the best of our knowledge. Further compounding the issue is that four variants of the Davey-Stewartson system exist, and that different authors write the system in different ways, which can often create confusion. The present work aims at addressing this issue and presenting the periodic, traveling wave solutions of all four variants of the Davey-Stewartson system in a concise but self-contained manner.

This work is organized as follows. In Section 2 we introduce the four variants of the Davey-Stewartson and we briefly review their Lax pair, invariances, and reductions, and one-dimensional reductions. In Section 3 we derive the periodic, traveling wave solutions of the defocusing DSII system. In Section 4 we present various examples, and in Section 5 we discuss various distinguished limits, including one-dimensional reductions, the plane-wave and soliton limits, and trivial-phase solutions. In Section 6 we generalize the calculations to all four variants of the Davey-Stewartson system, and in Section 7 we end this work with a few concluding remarks.

\section{Preliminaries: Davey-Stewartson Systems, Lax Pair, Symmetries and Reductions}

The four variants of the Davey-Stewartson system. The general Davey-Stewartson equations are the system

$$
\begin{aligned}
& i q_{t}+\frac{1}{2}\left(q_{x x}-\sigma q_{y y}\right)+\psi q=0 \\
& \psi_{x x}+\sigma \psi_{y y}=-v\left(|q|^{2}\right)_{x x}+\sigma v\left(|q|^{2}\right)_{y y}
\end{aligned}
$$

for a complex-valued function $q$ and a real-valued function $\psi$ of $x, y$ and $t$. The parameters $\sigma= \pm 1$ and $v= \pm 1$ determine the four possible variants of the system. Specifically, the values $\sigma=-1$ and $\sigma=1$ denote respectively the so-called Davey-Stewartson I (DSI) and the Davey-Stewartson II (DSII) systems. Likewise, the values $v=-1$ and $v=1$ identify the focusing and defocusing cases, although in this case the distinction is more ambiguous, since in this case one has focusing or defocusing behavior depending the particular spatial reduction is considered (see below for further details). For convenience, we list the four variants of the Davey-Stewartson system explicitly: 
(i) Focusing DSI equation $(\sigma=-1$ and $v=-1)$ :

$$
\begin{aligned}
& i q_{t}-\frac{1}{2}\left(q_{x x}+q_{y y}\right)+\psi q=0, \\
& \psi_{x x}-\psi_{y y}=\left(|q|^{2}\right)_{x x}+\left(|q|^{2}\right)_{y y} .
\end{aligned}
$$

(ii) Defocusing DSI equation $(\sigma=-1$ and $v=1)$ :

$$
\begin{aligned}
& i q_{t}-\frac{1}{2}\left(q_{x x}+q_{y y}\right)+\psi q=0, \\
& \psi_{x x}-\psi_{y y}=-\left(|q|^{2}\right)_{x x}-\left(|q|^{2}\right)_{y y} .
\end{aligned}
$$

(iii) Focusing DSII equation ( $\sigma=1$ and $v=-1)$ :

$$
\begin{aligned}
& i q_{t}+\frac{1}{2}\left(q_{x x}-q_{y y}\right)+\psi q=0 \\
& \psi_{x x}+\psi_{y y}=\left(|q|^{2}\right)_{x x}-\left(|q|^{2}\right)_{y y} .
\end{aligned}
$$

(iv) Defocusing DSII equation $(\sigma=v=1)$ :

$$
\begin{aligned}
& i q_{t}+\frac{1}{2}\left(q_{x x}-q_{y y}\right)+\psi q=0, \\
& \psi_{x x}+\psi_{y y}=-\left(|q|^{2}\right)_{x x}+\left(|q|^{2}\right)_{y y} .
\end{aligned}
$$

Note that the Davey-Stewartson equations appear written in many different ways in the literature. For example, the equations are often written using the auxiliary field - cf. [6],

$$
p(x, y, t)=\psi(x, y, t)-v|q(x, y, t)|^{2}
$$

instead of $\psi$, which we will also employ later. Different sign choices for the dependent and independent variables are also quite common. It might therefore be useful to briefly review how the above four versions are obtained from their Lax pair, since this might make it easier to switch from the present normalization choice to others. We do so next.

Lax pair. The Davey-Stewartson system (2.1) is the reduction $r(x, y, t)=v q^{*}(x, y, t)$ of the following more general system:

$$
\begin{gathered}
i q_{t}+\frac{1}{2}\left(q_{x x}-\sigma_{o}^{2} q_{y y}\right)+\psi q=0, \\
-i r_{t}+\frac{1}{2}\left(r_{x x}-\sigma_{o}^{2} r_{y y}\right)+\psi r=0, \\
\psi_{x x}+\sigma_{o}^{2} \psi_{y y}=-(q r)_{x x}+\sigma_{o}^{2}(q r)_{y y},
\end{gathered}
$$


where the star denotes complex conjugation and $\sigma=\sigma_{o}^{2}$, so that the DSI system corresponds to $\sigma_{o}=i$ and the DSII system to $\sigma_{o}=1$, respectively. We next show that the Lax pair for the system (2.3) is given by

$$
\begin{aligned}
& \mathbf{v}_{x}=Q \mathbf{v}-i \sigma_{o} J \mathbf{v}_{y}, \\
& \mathbf{v}_{t}=A \mathbf{v}+\sigma_{o}\left(Q \mathbf{v}_{y}-i \sigma_{o} J \mathbf{v}_{y y}\right),
\end{aligned}
$$

where

$$
J=\left(\begin{array}{cc}
1 & 0 \\
0 & -1
\end{array}\right), \quad Q(x, y, t)=\left(\begin{array}{ll}
0 & q \\
r & 0
\end{array}\right), \quad A(x, y, t)=\left(\begin{array}{ll}
a_{11} & a_{12} \\
a_{21} & a_{22}
\end{array}\right)
$$

and with

$$
\begin{aligned}
& \psi=i\left(a_{22}-a_{11}\right), \\
& \left(\partial_{x}+i \sigma_{o} J \partial_{y}\right) A_{d}=-\frac{1}{2} i J\left(\partial_{x}-i \sigma_{o} J \partial_{y}\right)(q r) .
\end{aligned}
$$

Above and below, the matrices $A_{d}$ and $A_{o}$ denote respectively the diagonal and off-diagonal parts of a matrix $A=A_{d}+A_{o}$. That is, we show that (2.3) is equivalent to the compatibility condition $\mathbf{v}_{t x}=\mathbf{v}_{x t}$ of (2.4), together with the constraints (2.5a) and (2.5b). (Note that one does not have the freedom to assign $A_{o}$, since $A_{o}$ is already determined by the requirement that the two parts of the Lax pair be compatible, see (2.7) below.)

It is immediate to verify that (2.3c) follows from (2.5a) and (2.5b). We must therefore show that (2.3a) and (2.3b) are equivalent to the constraint $\mathbf{v}_{t x}=\mathbf{v}_{x t}$. To this end, note that

$$
\left[Q, A_{d}\right]=\left(a_{22}-a_{11}\right) J Q, \quad\left[Q, A_{o}\right]=\left(a_{21} q-a_{12} r\right) J,
$$

where $[M, N]=M N-N M$ is the matrix commutator. Direct computation yields

$$
\begin{aligned}
& \mathbf{v}_{x t}=\left(Q_{t}+Q A-i \sigma_{o} J A_{y}\right) \mathbf{v}+\sigma_{o}\left(Q^{2}-i \sigma_{o} J Q_{y}-i J A\right) \mathbf{v}_{y}-\sigma_{o}^{3} \mathbf{v}_{y y y}, \\
& \mathbf{v}_{t x}=\left(A_{x}+A Q+\sigma_{o} Q Q_{y}-i \sigma_{0}^{2} J Q_{y y}\right) \mathbf{v}+\sigma_{o}\left(Q_{x}+Q^{2}-i A J-2 i \sigma_{o} J Q_{y}\right) \mathbf{v}_{y}-\sigma_{o}^{3} \mathbf{v}_{y y y} .
\end{aligned}
$$

The compatibility condition $\mathbf{v}_{x t}=\mathbf{v}_{t x}$ is therefore equivalent to the two equations

$$
\begin{aligned}
& i Q_{t}+i[Q, A]-i\left(\partial_{x}+i \sigma_{o} J \partial_{y}\right) A-i \sigma_{o} Q Q_{y}-\sigma_{o}^{2} J Q_{y y}=0, \\
& {[A, J]+i\left(\partial_{x}-i \sigma_{o} J \partial_{y}\right) Q=0 .}
\end{aligned}
$$

(Note that terms containing higher derivatives of $\mathbf{v}$ cancel automatically.) Since $[A, J]=$ $-2 J A_{o}$, it then follows from (2.6b) that

$$
A_{o}=\frac{1}{2} i J\left(\partial_{x}-i \sigma_{o} J \partial_{y}\right) Q
$$

which shows that, as anticipated, $A_{o}$ is not an independent quantity. In turn, using (2.7), we have

$$
\left[Q, A_{o}\right]=\frac{1}{2}\left(\sigma_{o}\left(q r_{y}-q_{y} r\right)-i(q r)_{x}\right) J .
$$


Splitting (2.6a) into its diagonal and off-diagonal parts then yields

$$
\begin{aligned}
& \frac{1}{2}\left(i \sigma_{o}\left(q r_{y}-q_{y} r\right)+(q r)_{x}\right) J-i\left(\partial_{x}+i \sigma_{o} J \partial_{y}\right) A_{d}-i \sigma_{o} Q Q_{y}=0, \\
& i Q_{t}+i\left(a_{22}-a_{11}\right) J Q-i\left(\partial_{x}+i \sigma_{o} J \partial_{y}\right) A_{o}-\sigma_{o}^{2} J Q_{y y}=0 .
\end{aligned}
$$

One can now verify that the left-hand-side of (2.8a) is equivalent to (2.5b). Similarly, using (2.5a) and (2.7) one can simplify (2.8b) to yield

$$
i J Q_{t}+\psi Q+\frac{1}{2}\left(\partial_{x x}-\sigma_{o}^{2} \partial_{y y}\right) Q=0,
$$

whose off-diagonal entries are precisely (2.3a) and (2.3b).

Symmetries. All four cases of the DS equations are invariant under a number of transformations. Specifically, if $q(x, y, t)$ and $\psi(x, y, t)$ are any solutions of $(3.1)$, so are the following, where all the transformation parameters are assumed to be real:

1. Space-time translations:

$$
\begin{aligned}
& \tilde{q}(x, y, t)=q\left(x-x_{0}, y-y_{0}, t-t_{0}\right), \\
& \tilde{\psi}(x, y, t)=\psi\left(x-x_{0}, y-y_{0}, t-t_{0}\right) .
\end{aligned}
$$

2. Galilean transformations:

$$
\begin{aligned}
& \tilde{q}(x, y, t)=q(x-k t, y+\sigma l t, t) \mathrm{e}^{i\left(k x+l y-\frac{1}{2}\left(k^{2}-\sigma l^{2}\right) t\right)}, \\
& \tilde{\psi}(x, y, t)=\psi(x-k t, y+\sigma l t, t) .
\end{aligned}
$$

3. Phase rotations:

$$
\begin{aligned}
& \tilde{q}(x, y, t)=q(x, y, t) \mathrm{e}^{i C t}, \\
& \tilde{\psi}(x, y, t)=\psi(x, y, t)+C .
\end{aligned}
$$

4. Scaling:

$$
\begin{aligned}
& \tilde{q}(x, y, t)=a q\left(a x, a y, a^{2} t\right), \\
& \tilde{\psi}(x, y, t)=a^{2} \psi\left(a x, a y, a^{2} t\right) .
\end{aligned}
$$

The above symmetries will allow us to identify the physical meaning of the parameters appearing in the periodic solutions. Importantly, however, unlike the two-dimensional NLS equation, none of the variants of the Davey-Stewartson system are rotationally invariant. 
One-dimensional reductions of the Davey-Stewartson system. The Davey-Stewartson system (2.3) admits self-consistent reductions to various one-dimensional systems, as we discuss next.

Let us first consider fields that are independent of $y$. In this case (2.3c) yields simply (for bounded solutions and up to phase rotations) $\psi=-q r$, and (2.3a) and (2.3b) become

$$
i q_{t}+\frac{1}{2} q_{x x}-q^{2} r=0, \quad-i r_{t}+\frac{1}{2} r_{x x}-r^{2} q=0 .
$$

The self-consistent reduction $r=v q^{*}$ then yields the one-dimensional defocusing and focusing nonlinear Schrödinger equation when $v=1$ and $v=-1$, respectively, as usual.

Now consider the reduction to fields that are independent of $x$. In this case (2.3c) yields $\psi=q r$, and now (2.3a) and (2.3b) become

$$
i q_{t}-\frac{1}{2} \sigma q_{y y}+q^{2} r=0, \quad-i r_{t}-\frac{1}{2} \sigma r_{y y}+r^{2} q=0 .
$$

Interesting, whether one gets the defocusing or the focusing NLS equation now also depends on the sign of $\sigma$, and not only on whether $r=q^{*}$ or $r=-q^{*}$. Specifically, let $r=v q^{*}$ with $v= \pm 1$ as before. When $\sigma=1$ the cases $v=1$ and $v=-1$ yield respectively the defocusing and focusing NLS equation, as before. However, when $\sigma=-1$ the situation is reversed, and $v=1$ and $v=-1$ yield respectively the focusing and defocusing NLS equation.

Periodic solutions of the $y$-independent reductions of the Davey-Stewartson system. As discussed above, when $q$ and $\psi$ are independent of $y$ both the DSI and the DSII systems reduce to the one-dimensional NLS equation. For later purposes, it is useful to briefly recall the periodic solutions of these one-dimensional systems.

Specifically, when $v=1$ and $q$ and $\psi$ are independent of $y$, both DSI and DSII reduce to the defocusing NLS equation

$$
i q_{t}+\frac{1}{2} q_{x x}-|q|^{2} q=0
$$

Eq. (2.11) has stationary solutions of the form — cf. [18],

$$
q(x, t)=R(x) \mathrm{e}^{i(\phi(x)-\omega t)},
$$

where the relevant functions and the corresponding parameters read

$$
\begin{array}{ll}
R^{2}(x)=A-m \mathrm{cn}^{2}(x, m), & \phi(x)=\int_{0}^{x} \frac{J_{1}}{R^{2}(s)} \mathrm{d} s, \\
\omega=\frac{1}{2}(3 A-2 m+1), & J_{1}^{2}=A(A-m)(A-m+1),
\end{array}
$$

together with the constraint $A \geq m$. Conversely, when $v=-1$, the $y$-independent reduction of the DSI and DSII system yields the focusing NLS equation

$$
i q_{t}+\frac{1}{2} q_{x x}+|q|^{2} q=0 .
$$


Stationary solutions of (2.14) in the form (2.12) yields (e.g., see [21])

$$
\begin{aligned}
& R^{2}(x)=A+m \mathrm{cn}^{2}(x, m), \quad \phi(x)=\int_{0}^{x} \frac{J_{1}}{R^{2}(s)} \mathrm{d} s, \\
& \omega=-\frac{1}{2}(3 A+2 m-1), \quad J_{1}^{2}=-A(A+m)(A+m-1)
\end{aligned}
$$

with the constraint $0 \leq A \leq 1-m$. We will recover all of the above solutions as reductions of the more general solutions of the focusing DSII system.

\section{Periodic Traveling Wave Solutions of the Defocusing DSII Equation}

For concreteness, we begin by considering the case of $\sigma=v=1$, namely, the defocusing DSII equation, of the general Davey-Stewartson system, which we now find it convenient to rewrite using the auxiliary field $p(x, y, t)$ introduced in (2.2) as

$$
\begin{aligned}
& i q_{t}+\frac{1}{2}\left(q_{x x}-\sigma q_{y y}\right)+\sigma\left(p+v|q|^{2}\right) q=0 \\
& p_{x x}+\sigma p_{y y}+2 v\left(|q|^{2}\right)_{x x}=0
\end{aligned}
$$

Later on we will see in Section 6 that similar calculations can be carried out for all four variants of the Davey-Stewartson system.

Stationary solutions. Taking advantage of the Galilean invariance of the Davey-Stewartson system, we first look for stationary solutions for the defocusing DSII equation, i.e., solutions for which both $|q|^{2}$ and $p$ are independent of time. That is, we look for solutions in the form

$$
\begin{aligned}
& q(x, y, t)=R(z) \mathrm{e}^{i(\phi(z)-\omega t)}, \\
& p(x, y, t)=P(z), \\
& z=a x+b y
\end{aligned}
$$

with $R, \phi$ and $P$ real functions of $z$ and $a, b$ and $\omega$ are real parameters. We also look for the functions $R$ and $P$ to be periodic.

Using the definitions of $q$ and $p$ in the form of (3.2), (3.1b) becomes $\left(a^{2}+b^{2}\right) P^{\prime \prime}+$ $2 a^{2}\left(R^{2}\right)^{\prime \prime}=0$, which in turn yields

$$
\left(a^{2}+b^{2}\right) P+2 a^{2} R^{2}=E_{1} z+E_{2} .
$$

Since we want the left-hand side of (3.3) to be periodic, we need to take $E_{1}=0$. Also, without loss of generality, the phase rotation symmetry allows us to neglect the constant $E_{2}$, or equivalently take $E_{2}=0$, thus obtaining simply

$$
P(z)=-\frac{2 a^{2}}{a^{2}+b^{2}} R^{2}(z)
$$


Note that $P$ is time-independent, as anticipated. Next, substituting (3.4) in (3.1a), using (3.2a) and separating the real and imaginary parts yields, respectively,

$$
\begin{aligned}
& \omega R+\frac{1}{2}\left(a^{2}-b^{2}\right)\left(R^{\prime \prime}-R\left(\phi^{\prime}\right)^{2}\right)-\frac{a^{2}-b^{2}}{a^{2}+b^{2}} R^{3}=0, \\
& R \phi^{\prime \prime}+2 R^{\prime} \phi^{\prime}=0 .
\end{aligned}
$$

Eq. (3.5b) can be solved immediately for $\phi^{\prime}$, to give

$$
\phi^{\prime}(z)=\frac{J_{1}}{R^{2}(z)},
$$

where $J_{1}$ is an arbitrary integration constant.

In turn, substituting (3.6) in (3.5a) reduces it to

$$
\omega R+\frac{1}{2}\left(a^{2}-b^{2}\right)\left(R^{\prime \prime}-\frac{J_{1}^{2}}{R^{3}}\right)-\frac{a^{2}-b^{2}}{a^{2}+b^{2}} R^{3}=0 .
$$

Multiplying by $2 R^{\prime}$, integrating,

$$
\left(a^{2}-b^{2}\right)\left(g^{\prime}\right)^{2}+4\left[\frac{b^{2}-a^{2}}{a^{2}+b^{2}} g^{3}+2 \omega g^{2}+2 J_{2} g-\left(a^{2}-b^{2}\right) J_{1}^{2}\right]=0,
$$

where $J_{2}$ is another arbitrary integration constant. Eq. (3.7b) is a differential equation of the form $\left(g^{\prime}\right)^{2}=4 P_{3}(g)$, where $P_{3}(\cdot)$ is a cubic polynomial. Looking for solutions in the form $g(z)=A+B Y^{2}$ maps (3.7b) into the ODE

$$
\left(Y^{\prime}\right)^{2}=\frac{c_{-2}}{Y^{2}}+c_{0}+c_{2} Y^{2}+c_{4} Y^{4}
$$

where the coefficients are

$$
\begin{aligned}
& c_{4}=\frac{B}{a^{2}+b^{2}}, \\
& c_{2}=\frac{3 A\left(a^{2}-b^{2}\right)-2 \omega\left(a^{2}+b^{2}\right)}{a^{4}-b^{4}}, \\
& c_{0}=\frac{3 A^{2}\left(a^{2}-b^{2}\right)-\left(4 \omega A+J_{2}\right)\left(a^{2}+b^{2}\right)}{\left(a^{4}-b^{4}\right) B}, \\
& c_{-2}=\frac{\left(a^{2}-b^{2}\right) A^{3}-\left(2 \omega A^{2}+J_{2} A\right)\left(a^{2}+b^{2}\right)-\left(a^{4}-b^{4}\right) J_{1}^{2}}{\left(a^{4}-b^{4}\right) B^{2}} .
\end{aligned}
$$

Imposing that (3.7c) matches the ODE satisfied by the Jacobian elliptic cosine [41], namely, $\left(Y^{\prime}\right)^{2}=\left(1-Y^{2}\right)\left(1-m+m Y^{2}\right)$, yields

$$
c_{-2}=0, \quad c_{0}=1-m, \quad c_{2}=2 m-1, \quad c_{4}=-m .
$$

This way we finally obtain

$$
R^{2}(z)=A+B \operatorname{cn}^{2}(z, m)
$$


where

$$
\begin{aligned}
B & =-m\left(a^{2}+b^{2}\right), \\
\omega & =\frac{\left(a^{2}-b^{2}\right)\left(3 A+(1-2 m)\left(a^{2}+b^{2}\right)\right)}{2\left(a^{2}+b^{2}\right)}, \\
J_{1}^{2} & =\frac{A\left(A-m\left(a^{2}+b^{2}\right)\right)\left(A+(1-m)\left(a^{2}+b^{2}\right)\right)}{a^{2}+b^{2}}, \\
J_{2} & =-\frac{a^{2}-b^{2}}{a^{2}+b^{2}}\left(3 A^{2}+2 A(1-2 m)\left(a^{2}+b^{2}\right)+m(m-1)\left(a^{2}+b^{2}\right)^{2}\right) .
\end{aligned}
$$

Therefore, the stationary solution (3.2) is completely determined by four parameters: $a, b, A$ and $m$. Importantly, however, self-consistency requires that some of the parameters must satisfy certain constraints. In particular, $J_{1}^{2}$ and $R^{2}(z)$ must be positive (cf. (3.6) and (3.10)). It is easy to see that both requirements are satisfied when

$$
A \geq m\left(a^{2}+b^{2}\right)
$$

It should be noted that (3.11c) leaves the sign of $J_{1}$ undetermined. However, both sign choices for $J_{1}$ yield valid solutions. This is because changing the sign for $J_{1}$ is equivalent to changing the sign of $z$ (as per (3.6), since (3.10) implies that $R(z)$ is even in $z$ ), and the Davey-Stewartson system is invariant under $x \mapsto-x$ and/or $y \mapsto-y$.

Traveling wave solutions. Next we use the invariances presented in Section 2 to generalize the above stationary solutions and obtain the most general periodic solutions of the defocusing DSII equation. Specifically, applying Galilean invariance (2.9) yields the following family of traveling wave solutions:

$$
\begin{aligned}
& q(x, y, t)=R(Z) \mathrm{e}^{i(\phi(Z)+k x+l y-\Omega t)}, \\
& p(x, y, t)=-\frac{2 a^{2}}{a^{2}+b^{2}} R^{2}(Z), \\
& Z(x, y, t)=a x+b y-(a k-b l) t,
\end{aligned}
$$

where

$$
\Omega=\omega+\frac{1}{2}\left(k^{2}-l^{2}\right),
$$

$\phi(\cdot)$ is still determined by (3.6), $R(\cdot)$ is still determined by (3.10), and the solution parameters are determined by (3.11) together with the constraint (3.12). We should note that, even though we used the symbol $Z$ for the similarity variable in (3.13c), for simplicity we will usually still denote it as $z$ below. The proper meaning should be clear from the context, and will anyhow be uniquely determined by the value of the parameters $k$ and $l$.

Summarizing, up to inessential phase rotations and space translations (which altogether would add three additional free parameters), the periodic solutions of the defocusing DSII equations are determined by six independent parameters: $a, b, k, l, A$ and $m$. Importantly, each of these parameters has a direct physical interpretation. Specifically: 
- $a$ and $b$ are the scaling parameters for the $x$ and $y$ coordinates, respectively. Namely, higher values of $a$ and/or $b$ yield thinner solutions with respect to $x$ and/or $y$.

- $k$ and $l$ are the wavenumbers for the $x$ and $y$ coordinates, respectively, which (by Galilean invariance) also determine the speed of propagation with respect to $x \& y$. As a special case, setting $k=0$ and/or $l=0$ yields solutions that are stationary with respect to $x$ and/or $y$.

- $A$ and $m$ determine the solution profile. Indeed, it should be obvious from (3.10) that the maximum and minimum values of $|q(x, y, t)|^{2}$ are respectively $A$ and $A-m\left(a^{2}+\right.$ $\left.b^{2}\right)$.

We will show below that, as with the focusing and defocusing NLS equations, the limiting case $m=0$ yields solutions with constant amplitude, whereas the limiting case $m=1$ yields the soliton solutions of the Davey-Stewartson system.

\section{Examples}

In this section and the following one, we elaborate on the traveling wave solutions $q(x, y, t)$ and $p(x, y, t)$ derived in Section 3. We begin by first presenting some concrete examples.

The profile of $|q(z)|$ as a function of $z$ only depends on $a^{2}+b^{2}$, not on the individual values of $a$ and $b$. Figs. 1 and 2 show plots of $|q|$ and $p$ as a function of $z$ for $k=l=0$ and different values of $m$ and different values of $a$ and $b$ satisfying the constraint $a^{2}+b^{2}=1$. With these choices, (3.13a) and (3.13b) yield

$$
|q(z)|^{2}=A-m \mathrm{cn}(z, m)^{2}, \quad p(z)=-\frac{2 a^{2}}{a^{2}+b^{2}}\left(A-m \mathrm{cn}(z, m)^{2}\right) .
$$

In the limit $m=0$, both $|q|$ and $p$ reduce to a constant, whereas, in the opposite limit $m=1$, one obtains the familiar cusp-shaped profile of the black solitons of the defocusing NLS equation. Both these limits are discussed in detail in Section 5, where we also show that, with the above parametrization, gray solitons are obtained when $A>1$. This is illustrated by Fig. 2, which shows the same kind of plots as in Fig. 2 but now with $A=2$.

While choosing different values of $a$ and $b$ subject to $a^{2}+b^{2}=1$ leaves $|q(z)|$ unaffected, it is important to realize, however, that doing so does amount to a rescaling of $p(z)$, as is easily seen in (4.1). The most dramatic manifestation of this phenomenon occurs when $a=$ 0 and $b= \pm 1$, in which case $p(z)$ is identically zero. This is an important consequence of the Davey-Stewartson system not being invariant under rotations of the spatial coordinates.

Similarly, even though the value of $|q(z)|$ as a function of $z$ only depends on the value of $a^{2}+b^{2}$, the spatial profile of $|q(x, y, t)|$ as a function of $x$ and $y$ does depend on the individual values of $a$ and $b$. This phenomenon is clearly illustrated in Figs. 3, 4 and 5 which show plots of $|q(x, y, t)|$ and $p(x, y, t)$ as functions of $x$ and $y$ at $t=0$ for different choices of $a$ and $b$ corresponding to the profile for $|q(z)|$ shown in Fig. 1. (Recall that all the solutions presented in this work are traveling wave solutions; therefore their spatial profile 


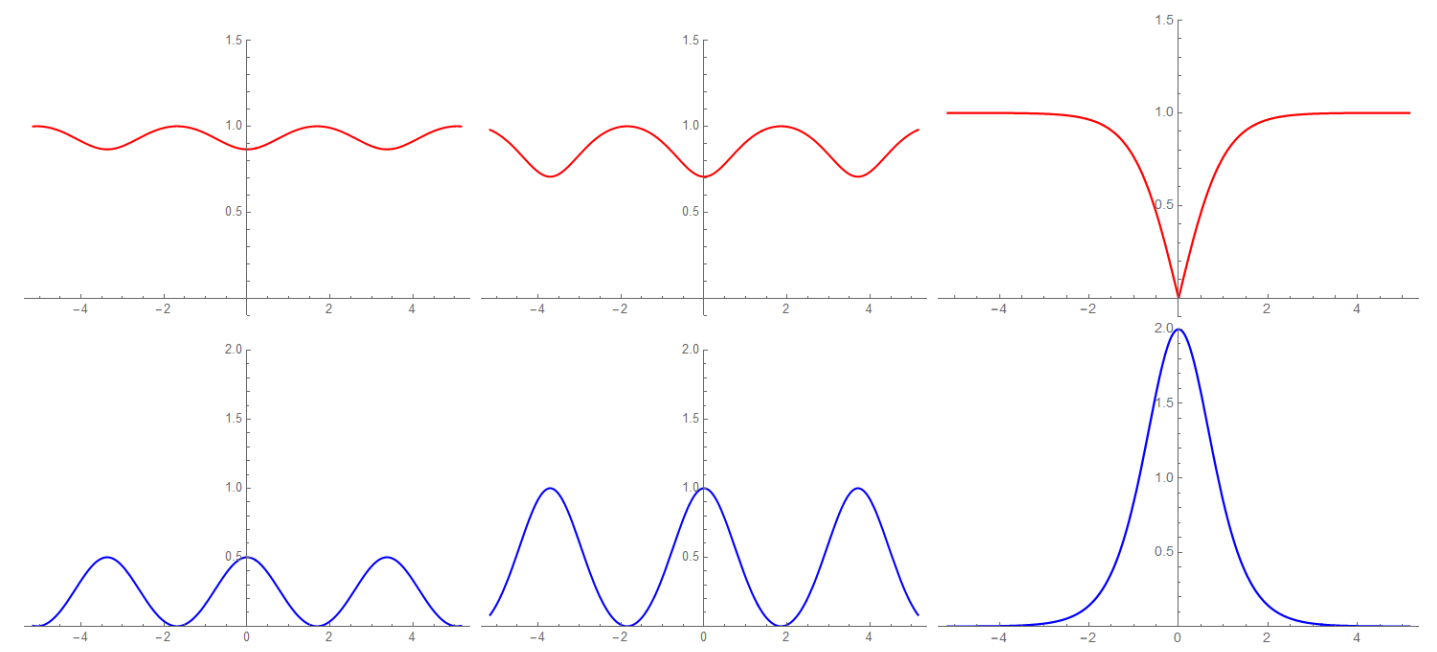

Figure 1: Plots of $|q(z)|$ (top row) and $p(z)$ (bottom row) in the reduction $A=1, a=1, b=0, k=l=0$. Left: $m=1 / 4$. Center: $m=1 / 2$. Right: $m=1$. Note $p(z)$ was shifted upward by 2 via (2.10) so that all its values are non-negative.

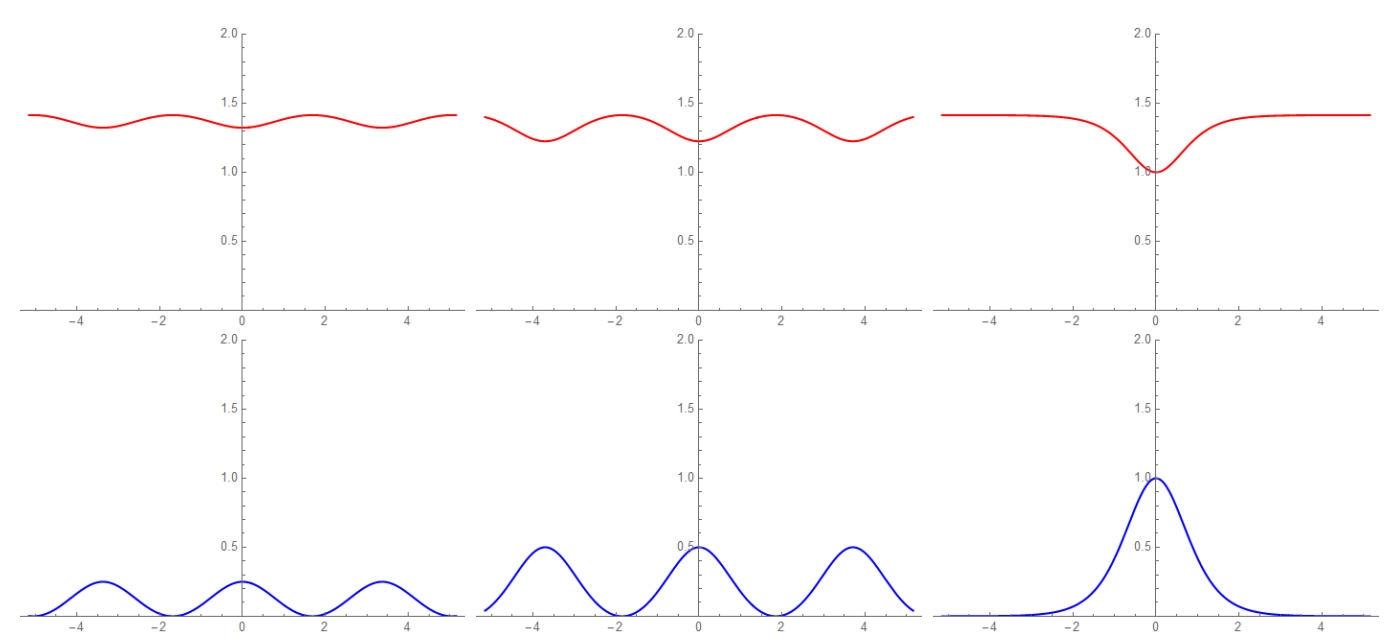

Figure 2: Same Fig. 1 , but now with $a=b=1 / \sqrt{2}$ and $A=2$.

is independent of $t$.) Similarly, Fig. 6 shows plots of $|q(x, y, t)|$ and $p(x, y, t)$ corresponding to the profile for $|q(z)|$ shown in Fig. 2.

\section{Special Cases, Reductions and Distinguished Limits}

We now discuss several reductions and distinguished limits of the general traveling wave solutions (3.13).

One-dimensional solutions. A special case of the traveling wave solutions (3.13) is that of effectively one-dimensional solutions, namely, solutions whose modulus is independent 

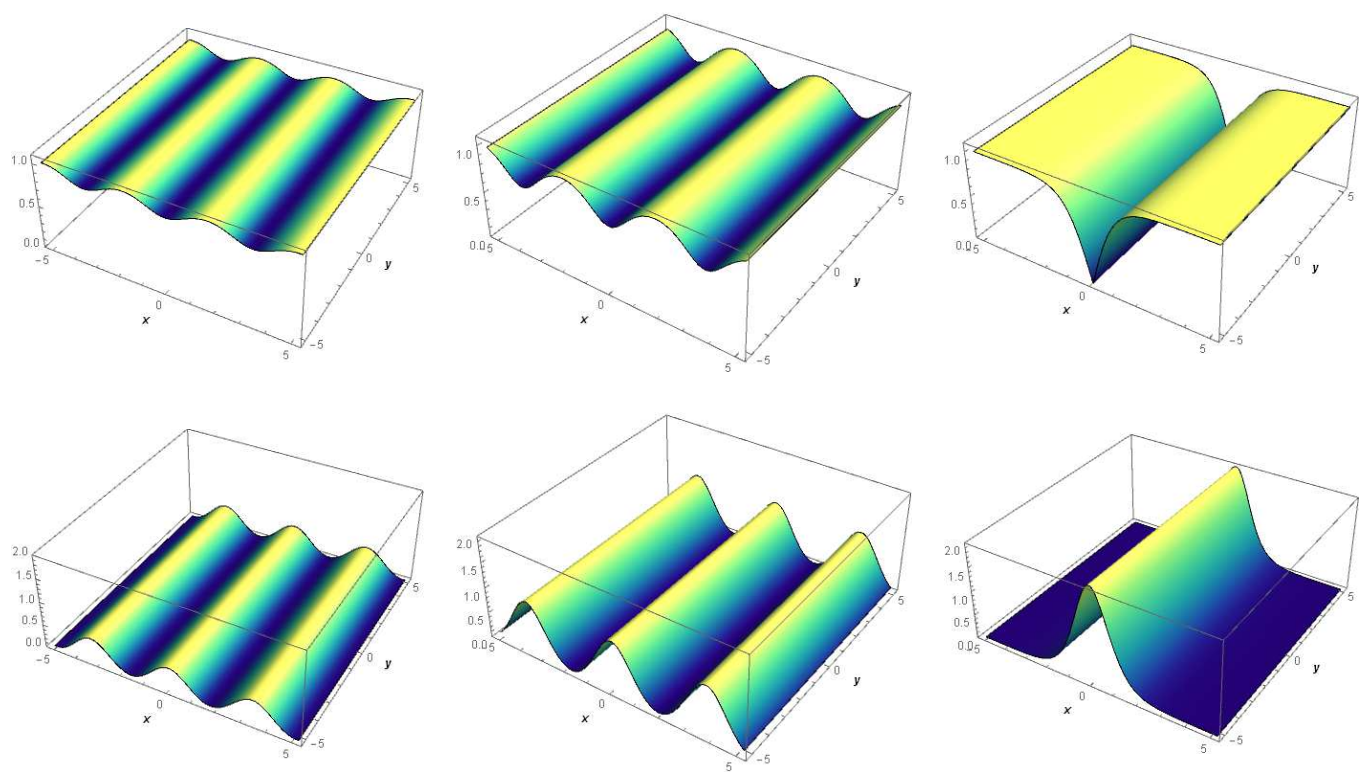

Figure 3: Plots of $|q(x, y, 0)|$ (top row) and $p(x, y, 0)$ (bottom row) in the reduction $A=1, k=l=0$, and with $(a, b)=(1,0)$. Left: $m=1 / 4$. Center: $m=1 / 2$. Right: $m=1$. Note $p(z)$ was shifted upward by 2 via (2.10) so that all its values are non-negative.
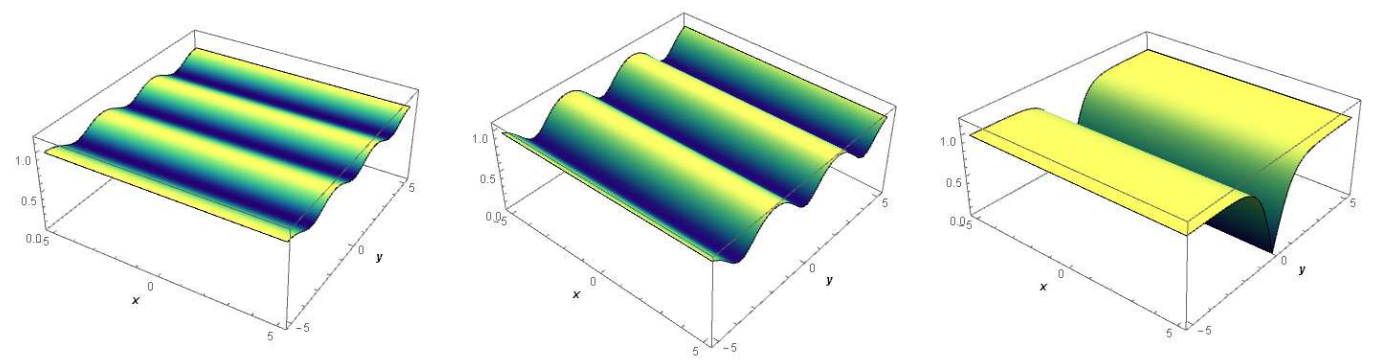

Figure 4: Same as Fig. 3, but with $(a, b)=(0,1)$. Since $p(x, y, t)$ is identically zero for all $(x, y, t)$ in this reduction, the corresponding plots have been omitted for brevity.

of either $x$ or $y$. Specifically, $|q(x, y, t)|$ is independent of $y$ when $b=0$. Note the expression for $q(x, y, t)$ is still $y$-dependent if $l \neq 0$. However, this $y$-dependence is trivial, as it is simply a linear exponential (corresponding to a plane wave), and one could also take $l=0$ without loss of generality thanks to Galilean invariance. In any case, (3.13) with $b=0$ yields, up to Galilean boosts in $y$, the general periodic solutions of the defocusing NLS equation (2.11) as

$$
q(x, y, t)=R(z) \mathrm{e}^{i(\phi(z)+k x+l y-\Omega t)},
$$

where $R(z)$ and $\phi(z)$ are given by Eqs. (3.10) and (3.6), respectively, with $z=a(x-k t)$ and $B, \Omega$ and $J_{1}$ as before. When $a=1$ and $k=0$, the above parameter dependencies reduce to (2.13). Also, in the limiting case $m=1$, the above yield the soliton solutions of the defocusing NLS equation as discussed in detail below. Similarly, solutions whose 

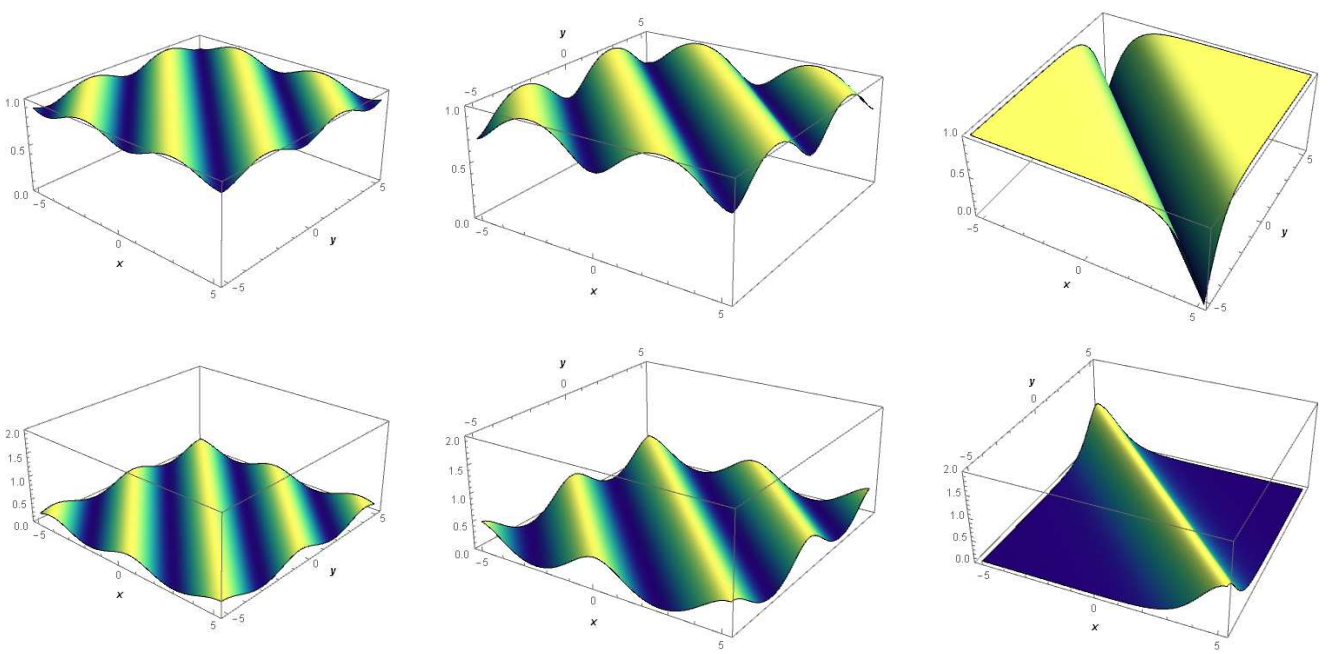

Figure 5: Same as Fig. 3, but with $(a, b)=(1 / \sqrt{2}, 1 / \sqrt{2})$. As in Fig. 2, the plots of $p(x, y, t)$ are shifted by 1 .
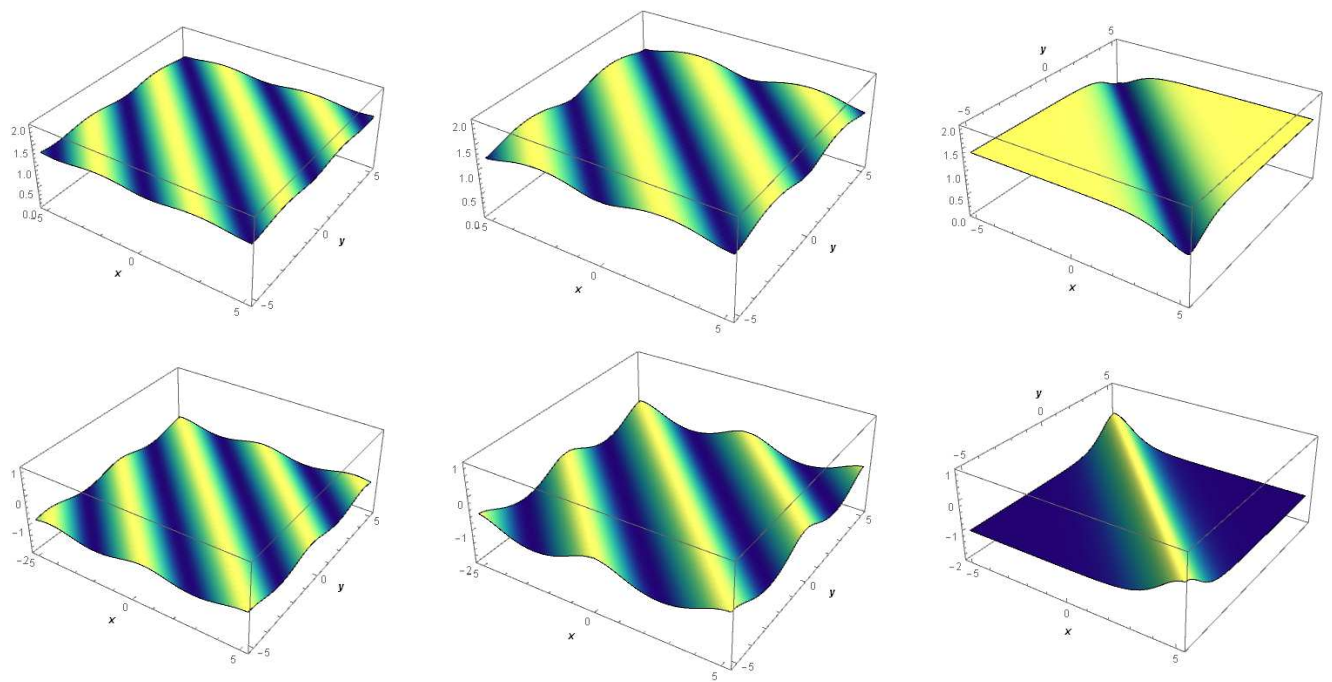

Figure 6: Same as Fig. 5, but with $A=2$. As in Fig. 2, the plots of $p(x, y, t)$ are shifted by 1.

modulus only depends on $y$ and $t$ are obtained when $a=0$, in which case the solution $q(y, t)$ is given by a similar expression as before, namely

$$
q(x, y, t)=R(z) \mathrm{e}^{i(\phi(z)+k x+l y-\Omega t)},
$$

where now $z=b(y+l t)$, and $B, \Omega$ and $J_{1}$ as before.

Trivial-phase solutions. Another distinguished limiting case is that of trivial-phase (or "flat-phase") solutions, which, as with the focusing and defocusing NLS equations [18,21], 
are obtained $J_{1}=0$. As can be seen from (3.11c) (and again similarly to the case of the defocusing NLS equations), for the defocusing DSII system there is only one family of flatphase solutions, which is obtained when

$$
A=m\left(a^{2}+b^{2}\right)
$$

in which case $\phi(Z)$ is identically zero (cf. (3.6)) and the solution reduces simply to

$$
q(x, y, t)=m \sqrt{a^{2}+b^{2}} \operatorname{sn}(Z, m) \mathrm{e}^{i(k x+l y-\Omega t)}
$$

with $Z$ and $\Omega$ given by (3.13) with (5.3).

Limit $m=0$, plane-wave solutions. Next we discuss the reductions of the periodic traveling wave solutions obtained in the limiting cases $m=0$ and $m=1$, starting with the former. When $m=0$, the radial part of the field $q(x, y, t)$ reduces simply to $R^{2}=A$. Neglecting the choice of sign (which can always be taken into account via spatial reflections) we then have

$$
\begin{aligned}
& q(x, y, t)=\sqrt{A} \mathrm{e}^{i\left(\frac{J_{1}}{A} z+k x+l y-\frac{1}{2}\left(k^{2}-l^{2}+2 \omega\right) t\right)}, \\
& p(x, y, t)=-\frac{2 a^{2}}{a^{2}+b^{2}} A,
\end{aligned}
$$

where $J_{1}$ and $\omega$ are given by (3.11) with $m=0$.

Limit $m=1$, soliton solutions. At the opposite limit, when $m=1$, one obtains the soliton solutions of the defocusing DSII system. For $m=1$, the radial part of (3.13) becomes

$$
R^{2}(z)=A-\left(a^{2}+b^{2}\right) \operatorname{sech}^{2}(z)
$$

where the constraint (3.12) reduces to

$$
A>a^{2}+b^{2}
$$

and the other solution parameters are

$$
\omega=\frac{1}{2} \frac{\left(a^{2}-b^{2}\right)\left(3 A-\left(a^{2}+b^{2}\right)\right)}{a^{2}+b^{2}}, \quad J_{1}=\delta A \sqrt{\frac{\left(A-\left(a^{2}+b^{2}\right)\right)}{a^{2}+b^{2}}},
$$

where $\delta= \pm 1$. (Recall that both signs of $\delta$ lead to acceptable solutions.) Integrating (3.6) with $m=1$ from 0 to $z$ and using (5.4), the phase $\phi(z)$ reduces to

$$
\phi(z)=\delta \sqrt{\frac{A-\left(a^{2}+b^{2}\right)}{a^{2}+b^{2}}} z+\arctan \left(\delta \sqrt{\frac{a^{2}+b^{2}}{A-\left(a^{2}+b^{2}\right)}} \tanh z\right) .
$$


Thus, putting everything together, we can write the soliton solutions of the defocusing DSII equation as

$$
\begin{aligned}
q(x, y, t)= & \left(\delta \sqrt{A-\left(a^{2}+b^{2}\right)}+i \sqrt{a^{2}+b^{2}} \tanh z\right) \\
& \times \exp \left[i\left(J_{1} z+k x+l y-\frac{1}{2}\left(k^{2}-l^{2}+2 \omega\right) t\right)\right], \\
p(x, y, t)= & -\frac{2 a^{2}}{a^{2}+b^{2}}\left(A-\left(a^{2}+b^{2}\right) \operatorname{sech}^{2} z\right)
\end{aligned}
$$

with

$$
z(x, y, t)=a x+b y-(a k-b l) t
$$

$J_{1}$ and $\omega$ given by (5.6), and where $A$ satisfies the constraint (5.5).

Reduction to the dark solitons of the defocusing NLS equation. It is instructive to see how, in the special case $b=l=0$, the soliton solutions (5.7) reduce to the well-known dark soliton solutions of the defocusing NLS equation up to Galilean transformations. Setting $b=l=0$ reduces the constraint (5.5) further to $A>a^{2}$. To ensure that the resulting solutions tend to constant values as $x \rightarrow \pm \infty$, we then set

$$
k=-\delta \operatorname{sgn}(a) \sqrt{A-a^{2}}
$$

in (5.7) (where the signum function $\operatorname{sgn}(a)$ takes value +1 for $a>0,-1$ for $a<0$ and 0 for $a=0$ ), which yields

$$
q(x, t)=\left(\delta \sqrt{A-a^{2}}+i|a| \tanh (z)\right) \mathrm{e}^{-i A t},
$$

where

$$
z=a\left(x+\delta \operatorname{sgn} a \sqrt{A-a^{2}} t\right) .
$$

Specifically, the asymptotic values of the solution (5.11) are

$$
q_{ \pm \infty}(t)=\lim _{x \rightarrow \pm \infty} q(x, t)=\left(\delta \sqrt{A-a^{2}} \pm i|a|\right) \mathrm{e}^{-i A t} .
$$

We can therefore rewrite (5.10) by introducing the angle $0 \leq \beta \leq \pi$ such that

$$
\cos \beta=\delta \sqrt{1-\frac{a^{2}}{A}}, \quad \sin \beta=\sqrt{\frac{a^{2}}{A}} .
$$

This allows us to write (5.10) in the familiar form of the dark solitons solutions of the defocusing NLS equation [57]

$$
q(x, t)=\sqrt{A}[\cos \beta+i \sin \beta \tanh (\sqrt{A}(x \sin \beta+t \cos \beta))] \mathrm{e}^{-i A t} .
$$


A wider class of stationary solutions. The stationary solutions derived in Section 3 correspond to setting $k=l=0$ in the general expression of the traveling wave solutions (3.13). A wider class of stationary solutions, however, can be obtained instead by enforcing the constraint

$$
a k-b l=0
$$

in (3.13), which allows one to eliminate one solution parameter. The possible cases can be classified as: (i) $k=0$, (ii) $l=0$, and (iii) $k \neq 0$ and $l \neq 0$. We next discuss each of these cases.

(i) If $k=0$, the constraint (5.12) implies $l=0$ or $b=0$. If $k=l=0$, the solutions reduce back to the stationary ansatz (3.2). If $k=b=0$, however, then $z=a x$, the constraint reads $A>m a^{2}$, and the periodic solutions (3.13) reduce to the $y$ independent solutions (5.1).

(ii) A similar situation is obtained if the constraint (5.12) is satisfied by taking $l=0$, in which case the periodic solutions (3.13) reduce to the $x$-independent solutions (5.2) (including the stationary solutions as a special case).

(iii) If $k \neq 0$ and $l \neq 0$, the stationary solutions are still given by the general traveling wave expression (3.13), but where $Z=a x+b y$ is now independent of time, and the constraint (5.12) allows one to eliminate one of the four parameters $a, b, k$ and $l$ in terms of the other three.

Summarizing, the defocusing DSII equation possesses a five-parameter class of stationary solutions, which generalizes the four-parameter class of solutions found earlier. (This situation should be compared to that of the defocusing NLS equation, which has a threeparameter family of stationary solutions, parameterized by $A, a$ and $m$ ).

\section{Periodic Traveling Wave Solutions of All Four Variants of the DS System}

We now extend the calculations of Section 3 to find periodic traveling wave solutions for all four Davey-Stewartson equations written in the form (2.3) using the modified auxiliary field $p(x, y, t)$ defined in (2.2). We begin as before from the ansatz (3.2) for the stationary solutions. Using the same steps as for the defocusing DSII equation, (3.1b) yields

$$
P(z)=-\frac{2 v a^{2}}{a^{2}+\sigma b^{2}} R^{2}(z) .
$$

Upon substitution into (3.1a), the imaginary part can be solved for $\phi^{\prime}(z)$ as before, to obtain

$$
\phi^{\prime}(z)=\frac{J_{1}}{R^{2}(z)}
$$

In turn, substituting (6.1) into (3.1a) yields an ODE which is then integrated once to obtain

$$
\frac{1}{2}\left(a^{2}-\sigma b^{2}\right)\left(R^{\prime}\right)^{2}+\frac{\left(a^{2}-\sigma b^{2}\right) J_{1}^{2}}{2 R^{2}}+J_{2}+\omega R^{2}-\frac{1}{2} v \frac{a^{2}-\sigma b^{2}}{a^{2}+\sigma b^{2}} R^{4}=0 .
$$


Multiplying this by $R^{2}$, setting $R^{2}=A+B Y^{2}$ and performing the same steps as in the defocusing DSII case now reduces the above equation to the same form as (3.7c), but where now

$$
\begin{aligned}
& c_{4}=\frac{B v}{a^{2}+\sigma b^{2}}, \quad c_{2}=\frac{3 A v\left(a^{2}-\sigma b^{2}\right)-2 \omega\left(a^{2}+\sigma b^{2}\right)}{\left(a^{2}-\sigma b^{2}\right)\left(a^{2}+\sigma b^{2}\right)}, \\
& c_{0}=\frac{3 v\left(a^{2}-\sigma b^{2}\right) A^{2}-4 \omega\left(a^{2}+\sigma b^{2}\right) A-2\left(a^{2}+\sigma b^{2}\right) J_{2}}{B\left(a^{2}-\sigma b^{2}\right)\left(a^{2}+\sigma b^{2}\right)}, \\
& c_{-2}=\frac{1}{B^{2}}\left(\frac{v\left(a^{2}-\sigma b^{2}\right) A^{3}-2 \omega\left(a^{2}+\sigma b^{2}\right) A^{2}-2 J_{2}\left(a^{2}+b^{2} \sigma\right) A}{\left(\sigma a^{2}-b^{2}\right)\left(a^{2}+b^{2} \sigma\right)}-J_{1}^{2}\right) .
\end{aligned}
$$

If $\sigma=v=1,(6.2)$ reduce to (3.8). By requiring that the ODE (3.7c) matches that for the elliptic cosine, as before, one obtains (3.10), and, solving for the parameters, (6.2) yield (3.11). In the other three cases, by applying the same approach of defocusing DSII, the above setting allows us to express the periodic solutions in all four cases in the same form as (3.10). By the same approach (and after some simplification) the general parameter dependencies read

$$
\begin{aligned}
& B=-m v\left(a^{2}+b^{2} \sigma\right), \\
& \omega=\frac{\left(\sigma a^{2}-b^{2}\right)\left(3 v A+(1-2 m)\left(a^{2}+\sigma b^{2}\right)\right)}{2\left(a^{2}+\sigma b^{2}\right)}, \\
& J_{1}^{2}=\frac{A v\left(A-m v\left(a^{2}+b^{2} \sigma\right)\right)\left(A+v(1-m)\left(a^{2}+b^{2} \sigma\right)\right)}{a^{2}+b^{2} \sigma}, \\
& J_{2}=-\frac{v\left(\sigma a^{2}-b^{2}\right)}{2\left(a^{2}+b^{2} \sigma\right)}\left(m(m-1)\left(a^{2}+b^{2} \sigma\right)^{2}-2 v(2 m-1)\left(a^{2}+b^{2} \sigma\right) A+3 A^{2}\right) .
\end{aligned}
$$

The above calculations show that, for all four variants of the Davey-Stewartson system, the stationary solutions are completely determined by four independent parameters $A, m, a, b$. The most significant difference between the various cases lies in the precise parameter dependence (as specified by the above equations) and in the parameter constraints. Specifically, as with the defocusing DSII equation, we have two such constraints: (i) $R^{2}(z)$ must be non-negative for all $z$, and (ii) $J_{1}^{2}$ must also be non-negative. If $\sigma=v=1$, these constraints obviously reduce to (3.12). Next we proceed to discuss the remaining three cases in detail, and we will see that in some cases, the constrains end up being considerably more complicated.

Focusing DSII. Here $\sigma=1 v=-1$, and the matching yields

$$
B=-m\left(a^{2}+b^{2}\right)
$$

with the constraint

$$
0 \leq A \leq(1-m)\left(a^{2}+b^{2}\right)
$$


Defocusing DSI. Here $\sigma=-1 v=1$, and the matching yields

$$
B=-m\left(a^{2}-b^{2}\right)
$$

with the constraints

$$
A-m\left(a^{2}-b^{2}\right) \geq 0, \quad \frac{A-m\left(a^{2}-b^{2}\right)}{a^{2}-b^{2}} \geq-1 .
$$

Focusing DSI. Here $\sigma=-1 v=-1$, and the matching yields

$$
B=m\left(a^{2}-b^{2}\right)
$$

with the constraints

$$
A+m\left(a^{2}-b^{2}\right) \geq 0, \quad \frac{A+m\left(a^{2}-b^{2}\right)}{a^{2}-b^{2}} \leq 1 .
$$

Traveling wave solutions, reductions and limiting cases. Now that the stationary solutions of all four variants of the Davey-Stewartson system have been found, the traveling wave solutions are generated by taking advantage of the Galilean invariance of the system, namely (2.9). Since the transformation is identical to the one presented in Section 3, we omit the resulting formulae. Also, one can study the reductions and limiting cases of these solutions in a similar way as for the defocusing DSII system. Again, since the calculations are very similar to those presented in Section 5, we omit them for brevity.

\section{Concluding Remarks}

In conclusions, we presented the full class of periodic solutions of all four versions of the Davey-Stewartson system, we illustrated these solutions with examples, and we discussed several distinguished limits, including one-dimensional, linear and solitonic reductions.

The results of this work should pave the way for further investigations on several different fronts. A first obvious question is that of the stability of all these periodic solutions. One can expect that establishing linear stability should be relatively straightforward, even though it involves the study of solutions of a linearized PDE, as opposed to an ODE. Indeed, since the periodic solutions are essentially one-dimensional, it should be possible to carry it out using similar techniques as for the KP equation $[40,55]$. On the other hand, the study of nonlinear stability might be considerably more difficult than for one-dimensional systems $[18,19,21]$.

Another possible avenue for further study will be the study of the semiclassical (i.e., small-dispersion) limit of the Davey-Stewartson system. Indeed, various studies have already revealed the emergence of nearly periodic oscillations over small spatial scales [12, 37], which indicates that periodic solutions will play a key role in the analysis.

Yet a further direction for further study will be the development of Whitham modulation theory $[24,51]$ for the Davey-Stewartson system, following the recent development of 
the Whitham modulation equations for the Kadomtsev-Petviashvili equation [4] and other $(2+1)$-dimensional equations of KP type $[3,5]$. We note that the availability of Whitham modulation theory has allowed researchers to effectively study a variety of phenomena, both for one-dimensional systems (e.g., see $[7,15,16,22-24,30,38]$ ) as well as for $(2+1)$ dimensional ones $[45,46]$. We hope that this will also prove to be the case for the DaveyStewartson systems.

\section{Acknowledgment}

This work was partially supported by the National Science Foundation under grant number DMS-2009487.

\section{References}

[1] M.J. Ablowitz, G. Biondini and S. Blair, Multi-dimensional pulse propagation in non-resonant $\chi^{(2)}$ materials, Phys. Lett. A 236, 520-524 (1997).

[2] M.J. Ablowitz, G. Biondini and S. Blair, Nonlinear Schrödinger equations with mean terms in non-resonant multi-dimensional quadratic materials, Phys. Rev. E 63, 046605 (2001).

[3] M.J. Ablowitz, G. Biondini and I. Rumanov, Whitham modulation theory for (2+1)-dimensional equations of Kadomtsev-Petviashvili type, J. Phys. A, 51, 215501 (2018).

[4] M.J. Ablowitz, G. Biondini and Q. Wang, Whitham modulation theory for the KadomtsevPetviashvili equation, Proc. Roy. Soc. London A, 473, 20160695 (2017).

[5] M.J. Ablowitz, G. Biondini and Q. Wang, Whitham modulation theory for the two-dimensional Benjamin-Ono equation, Physical Review E, 96, 032225 (2017).

[6] M.J. Ablowitz and P.A. Clarkson, Solitons, Nonlinear Evolution Equations and Inverse Scattering, Cambridge University Press (1991).

[7] M.J. Ablowitz, J.T. Cole and I. Rumanov, On the Whitham system for the radial nonlinear Schrödinger equation, Stud. Appl. Math. 142, 269-313 (2019).

[8] M.J. Ablowitz, A. Ramani and H. Segur, Nonlinear evolution equations and ordinary differential equations of Painlevé type, Lett. Nuovo Cimento 23, 333-338 (1978).

[9] M.J. Ablowitz, A. Ramani and H. Segur, A connection between nonlinear evolution equations and ordinary differential equations of P-type. I \& II, J. Math. Phys. 21, 715-721 (1980), J. Math. Phys. 21, 1006-1015 (1980).

[10] M.J. Ablowitz and H. Segur, On the evolution of packets of water waves, J. Fluid Mech. 92, 691 (1979).

[11] M.J. Ablowitz and H. Segur, Solitons and the inverse scattering transform, SIAM (1981).

[12] O. Assainova, C. Klein, K.D.T.-R. McLaughlin and P.D. Miller, A study of the direct spectral transform for the defocusing Davey-Stewartson II equation in the semiclassical limit, Commun. Pure Appl. Math. 72, 1474-1547 (2019).

[13] E.D Belokolos, A.I. Bobenko, V.Z. Enolskii, A.R. Its and V.B. Matveev, Algebro-Geometric Approach to Nonlinear Integrable Equations, Springer (1994).

[14] D.J. Benney and G.J. Roskes, Wave instabilities, Stud. Appl. Math. 48, 377 (1969).

[15] G. Biondini, Riemann problems and dispersive shocks in self-focusing media, Phys. Rev. E 98, 052220 (2018).

[16] G. Biondini and Y. Kodama, On the Whitham equations for the defocusing nonlinear Schrodinger equation with step initial data, J. Nonlin. Sci. 16, 435-481 (2006). 
[17] M. Boiti, J. Leon, L. Martina and F. Pempinelli, Scattering of localized solitons in the plane, Phys. Lett. A 132, 432-439 (1988).

[18] N. Bottman, B. Deconinck and M. Nivala, Elliptic solutions of the defocusing NLS equation are stable, J. Phys. A 44, 285201 (2011).

[19] J. Carter and H. Segur, Instabilities in the two-dimensional cubic nonlinear Schrödinger equation, Phys. Rev. E 68, 045601R (2003).

[20] A. Davey and K. Stewartson, On three-dimensional packets of surface waves, Proc. Roy. Soc. London A 338, 101-110 (1974).

[21] B. Deconinck and B.L. Segal, The stability spectrum for elliptic solutions to the focusing NLS equation, Phys. D 346, 1-19 (2017).

[22] G.A. El, V.V. Geogjaev, A.V. Gurevich and A.L. Krylov, Decay of an initial discontinuity in the defocusing NLS hydrodynamics, Phys. D 87, 186-192 (1995).

[23] G.A. El, A.V. Gurevich, V.V. Khodorovskii and A.L. Krylov, Modulational instability and formation of a nonlinear oscillatory structure in a focusing medium, Phys. Lett. A, 177, 357-361 (1993).

[24] G.A. El and M.A. Hoefer, Dispersive shock waves and modulation theory, Phys. D 333, 11-65 (2016).

[25] A.S. Fokas, Integrable multidimensional versions of the nonlocal nonlinear Schrödinger equation, Nonlinearity 29, 319 (2016).

[26] A.S. Fokas and M.J. Ablowitz, Method of solution for a class of multidimensional nonlinear evolution equations, Phys. Rev. Lett. 51, 7-10 (1983).

[27] A.S. Fokas and M.J. Ablowitz, On the inverse scattering transform of multidimensional nonlinear equations related to first-order systems in the plane, J. Math. Phys. 25, 2494-2505 (1984).

[28] A.S. Fokas and P.M. Santini, Dromions and a boundary value problem for the Davey-Stewartson 1 equation, Phys. D 44, 99-130 (1990).

[29] F. Gungor and C. Ozemir, Dispersionless Davey-Stewartson system: Lie symmetry algebra, symmetry group and exact solutions, arXiv:2012.10664.

[30] M.A. Hoefer, M.J. Ablowitz, I. Coddington, E. Cornell, P. Engels and V. Schweikhard, Dispersive and classical shock waves in Bose-Einstein condensates and gas dynamics, Phys. Rev. A 74, 023623 (2006).

[31] E. Infeld and G. Rowlands, Nonlinear Waves, Solitons And Chaos, Cambridge University Press (2000).

[32] A.R. Its and V.P. Kotlyarov, Explicit formulas for solutions of the Schrödinger nonlinear equation, Dokl. Akad. Nauk USSR A 11, 965 (1976).

[33] A.M. Kamchatnov, On improving the effectiveness of periodic solutions of the NLS and DNLS equations, J. Phys. A 23, 2945-2960 (1990).

[34] A.M. Kamchatnov, Nonlinear periodic waves and their modulations, World Scientific (2000).

[35] P. Kevrekidis, D. Frantzeskakis, R. Carretero-Gonzalez, Emergent Nonlinear Phenomena in BoseEinstein Condensates: Theory and Experiment, Springer (2007).

[36] C. Klein and K.D.T.-R. McLaughlin, Spectral approach to D-bar problems, Comm. Pure Appl. Math. 70, 1052-1083 (2017).

[37] C. Klein and K. Roidot, Numerical study of the semiclassical limit of the Davey-Stewartson II equations, Nonlinearity 27, 2177-2214 (2014).

[38] Y. Kodama, The Whitham equations for optical communications: mathematical theory of NRZ, SIAM J. Appl. Math. 59, 2162-2192 (1999).

[39] A.C. Newell and J.V. Moloney, Nonlinear Optics, Academic Press (1992).

[40] D.E. Pelinovsky and Y.A. Stepanyants, Self-focusing instability of plane solitons and chains of two-dimensional solitons in positive-dispersion media, Sov. Phys. JETP 77, 602-608 (1993).

[41] F.W.J. Olver et alii, NIST Handbook of Mathematical Functions, Cambridge (2010). 
[42] P.A. Perry, Global well-posedness and long-time asymptotics for the defocussing Davey-Stewartson II equation in $H^{1 ; 1}(\mathbb{C})$, J. Spectr. Theory 6, 429-481 (2016).

[43] J. Rao, Y. Cheng, K. Porsezian, D. Mihalache and J. He, PT-symmetric nonlocal Davey-Stewartson I equation: soliton solutions with nonzero background, Physica D 401, 132180 (2020).

[44] J. Rao, J. He, D. Mihalache and Y. Cheng, PT-symmetric nonlocal Davey-Stewartson I equation: general lump-soliton solutions on a background of periodic line waves, Appl. Math. Lett. 104, 106246 (2020).

[45] S. Ryskamp, M.A. Hoefer and G. Biondini, Oblique interactions between solitons and mean flow in the Kadomtsev-Petviashvili equation, Nonlinearity 34, 3583 (2021).

[46] S. Ryskamp, M. Maiden, G. Biondini and M. A. Hoefer, Evolution of truncated and bent wave solitons: the Mach expansion problem, J. Fluid Mech. 909, A24 (2021).

[47] H. Segur and M.J. Ablowitz, Asymptotic solutions of nonlinear evolution equations and a Painlevé transcedent, Phys. D 3, 165-184 (1981).

[48] M. Tajiri and T. Arai, Periodic soliton solutions of the Davey-Stewartson equation, Proc. Inst. Math. Nat. Acad. Sci Ukraine 30, 210-217 (2000).

[49] M. Tajiri and M. Hagiwara, Similarity solutions of the two-dimensional coupled nonlinear Schrödinger equation, J. Phys. Soc. Japan 52, 3727-3734 (1983).

[50] T. Xu, M. Li, Y. Huang, Y. Chen and C. Yu, Nonsingular localized wave solutions for the nonlocal Davey-Stewartson I equation with zero background, Mod. Phys. Lett. B 35, 1750338 (2017).

[51] G.B. Whitham, Linear and Nonlinear Waves, Wiley (1974).

[52] G. Yi, On the dispersionless Davey-Stewartson system: Hamiltonian vector field Lax pair and relevant nonlinear Riemann-Hilbert problem for dDS-II system, Lett. Math. Phys. 110, 445-463 (2020).

[53] G. Yi, On the dispersionless Davey-Stewartson hierarchy: Zakharov-Shabat equations, twistor structure and Lax-Sato formalism, arXiv:1812.10220v3 (2020).

[54] V.E. Zakharov, Collapse of Langmuir waves, Sov. Phys. JETP 35, 908 (1972).

[55] V.E. Zakharov, Instability and nonlinear oscillations of solitons, JETP Lett. 22, 174-179 (1975).

[56] V.E. Zakharov and A.B. Shabat, Exact theory of two-dimensional self-focusing and one-dimensional self-modulation of waves in nonlinear media, Sov. Phys. JETP 34, 62-60 (1972).

[57] V.E. Zakharov and A.B. Shabat, Interaction between solitons in a stable medium, Sov. Phys. JETP 37, 823-828 (1973). 